\title{
The Influence of Weight Distribution on the Handling Characteristics of Intercity Bus under Steady State Vehicle Cornering Condition
}

\author{
Ekalak Prompakdee ${ }^{1}$, Pornporm Boonporm ${ }^{2}$ and Supakit Rooppakhun ${ }^{2}$ \\ ${ }^{1}$ School of Manufacturing Engineering, Institute of Engineering, Suranaree University of Technology, Nakhonratchasima, 30000 Thailand. \\ ${ }^{2}$ School of Mechanical Engineering, Institute of Engineering, Suranaree University of Technology, Nakhonratchasima, 30000 Thailand.
}

\begin{abstract}
The vehicle cornering behavior is an important performance in handling stability especially for the intercity bus. Accordingly, one of the significant parameters concerns the weight distribution which is affected by the center of gravity (CG). In this paper, the effect of weight distribution while varying the turning radius is compared and it should be expressed, and interpreted by understeer gradient which is influenced by the location of CG. The characteristic of intercity bus was modeled and evaluated using the multi-body dynamic analysis software. The analysis has been conducted under steady state cornering based on a total of three configurations, with front/rear axle weight in percent, as $40 / 60,45 / 55$, and 50/50. The results stated that the magnitude of weight distribution on front axle of bus in a range of $40 \%$ to $50 \%$ caused the incremental value of understeer gradient and it was also increased as three times in each turning radius since the difference lateral slip angles between front and rear are expanded.
\end{abstract}

\section{Introduction}

Nowadays, travelling by means of intercity bus in Thailand plays an extremely important role. From the department of land transport statistics, there are more than 35 million passengers for the intercity bus passenger [1]. Consequently, the number of intercity bus accidents should be taken into consideration and the root causes came from its yaw stability during cornering. It required both static and dynamic tests for this safety issue [2]. The static stability test related the vehicle's rollover resistance is a calculation of the center of gravity that affects from the significant parameters of vehicle weight distribution such as the position of engine, transmission, axle and other component $[3,4]$. In case of dynamics, the subject deals with the study of vehicle response related to driver input, ability of the vehicle to stabilize its motion against external disturbances and ride characteristics of vehicle [5-7]. The vehicle handling performance including the stability control were the crucial factors involved to the standard tests consisted of lateral acceleration, roll moment, braking performance, and CG position [8,9].

Generally, the most autonomies safety systems such as antilock braking, traction control and stability control have been introduced in passenger vehicles but in such systems like heavy vehicle (e.g., truck and bus) requires a sound knowledge of the steering response characteristics under both steady-state and transient conditions as well as handling performance [10]. However, the research related to the study of dynamics response during cornering of the heavy vehicle.
Therefore, the study aimed to evaluate the handling characteristics of a typical intercity bus under steady-state cornering condition. Using the multi-body dynamic model and simulation, the effect of weight distribution including various turning radius of intercity bus to handling characteristics (e.g. lateral acceleration, slip angle, and understeer gradient) were conducted. The understeer gradient can be evaluated by operating the vehicle around a constant radius turn and observing steering angle versus lateral acceleration.

\section{Steady state vehicle cornering}

The well-known bicycle model is considered in order to identify the relationship among the various parameters like lateral, longitudinal as well as yaw dynamics. In figure $1, \mathrm{X}-\mathrm{Y}$ earth fixed coordinate frame is defined in direction like east and north and $x-y-z$ rotating frame at the vehicle's center of gravity. Assuming plane kinematics, the rotation angle about vertical axis, called yaw angle $(\psi)$, is taken into account.

To define the relationship between the fix frame X-Y and the rotating frame $\mathrm{x}-\mathrm{y}$, the acceleration analysis is perform. Let $r=-R \hat{j}$, the acceleration of rigid body is defined as

$\vec{a}=\dot{\psi} \hat{k} x(\dot{\psi} \hat{k} x(-R \hat{j}))+\ddot{\psi} \hat{k} x(-R \hat{j})+2 \dot{\psi} \hat{k} x(-\dot{R} \hat{j})+\ddot{x} \hat{i}+\ddot{y} \hat{j}$

So that the lateral acceleration is

$$
\vec{a}_{y}=\dot{\psi}^{2} R+\ddot{y}
$$


The term $\ddot{y}$ is interesting because it shows the vehicle slip so that side slip behavior cannot be negligible

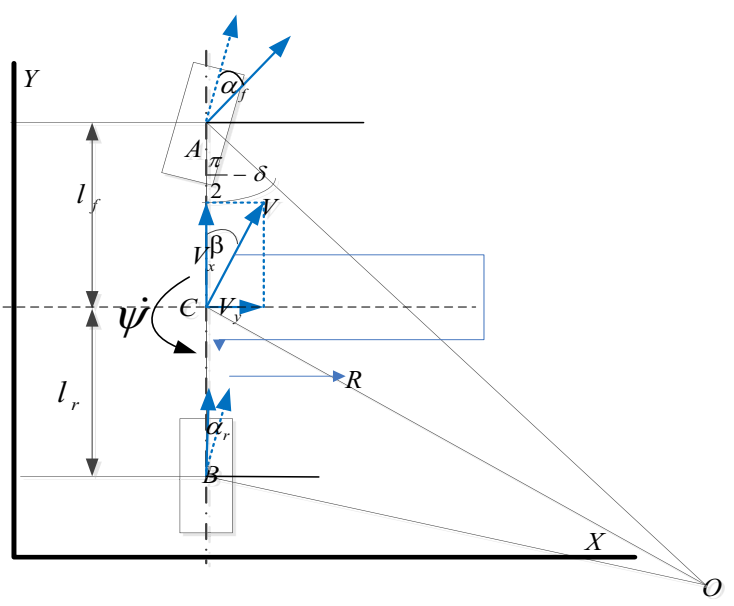

Figure 1. Steady state vehicle cornering condition.

Steady state cornering is the practical test in order to verify the handling characteristics. The aim of this test is to determine the understeer gradient of difference configuration of weigh transfer. The front and rear wheel slip angle denote by $\alpha_{f}$ and $\alpha_{r}$, respectively as shown in figure 1. The instantaneous turn center $O$ of the vehicle is the point at which the two lines perpendicular to the velocities of the two wheels meet. Under the assumption that the road radius is much larger than the wheel base of the vehicle. From the geometry, we have the relationship of the steering angle $\delta$ that can be approximated as

$$
\delta-\alpha_{f}-\alpha_{r} \approx \frac{L}{R}
$$

Consider the dynamics equations, $\alpha_{f}$ and $\alpha_{r}$ are related to the forces acted on tired and the small slip is assumed so that the lateral tired force is proportional to its slip angle with the constant called cornering stiffness for front $C_{f}$ and rear $C_{r}$

The steady state steering angle in equation 3 can be rewritten to

$$
\delta=\frac{L}{R}+\alpha_{f}-\alpha_{r}=\frac{L}{R}+\left(\frac{m_{f}}{2 C_{f}}-\frac{m_{r}}{2 C_{r}}\right) \frac{V_{x}^{2}}{R}
$$

or

$$
\delta=\frac{L}{R}+K_{v} a_{y}
$$

In this case of understeer, the understeer gradient $K_{v}$ should greater than zero due to a larger slip angle at the front tires compared to the rear tires. The relationship between weight transfer and wheel slip angle is interpreted in following equation 6 .

$$
\frac{m_{f}}{C_{f}}>\frac{m_{r}}{C_{r}} \Rightarrow \alpha_{f}>\alpha_{r}
$$

\section{Multi-body dynamic simulation}

The basic information required to understand multi-body dynamic simulation is its mathematical formulation. It also explains how lateral forces are generated while a vehicle turning, and their relation with the slip angle and lateral acceleration as well as understeer gradient. The intercity bus model is used to conduct for estimating vehicle handling characteristic as shown in Figure 2.

\begin{tabular}{|c|c|c|}
\hline \multicolumn{2}{|c|}{ Parameters } & Bus model \\
\hline \multicolumn{2}{|c|}{ Gross Vehicle Weight } & $1,8000 \mathrm{~kg}$ \\
\hline \multicolumn{2}{|c|}{ Overall Length } & $12,000 \mathrm{~mm}$ \\
\hline \multicolumn{2}{|c|}{ Wheelbase } & $5,139 \mathrm{~mm}$ \\
\hline \multirow{2}{*}{ Track Width } & Front & $2,120 \mathrm{~mm}$ \\
\hline & Rear & $1,780 \mathrm{~mm}$ \\
\hline \multirow{2}{*}{ Suspension } & Front & Coil Spring \\
\hline & Rear & Coil Spring \\
\hline \multicolumn{2}{|c|}{ Front Axle } & Double wishbone \\
\hline \multicolumn{2}{|c|}{ Rear Axle } & Rigid axle \\
\hline \multicolumn{2}{|c|}{ Tires } & $318 / 80$ R22.5 \\
\hline
\end{tabular}

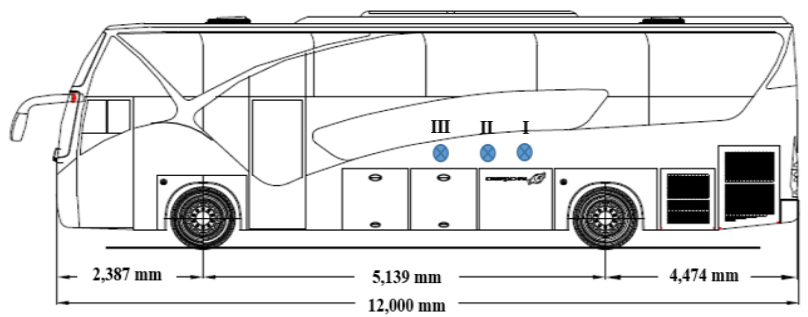

Figure 2. Location of Center of Gravity (CG.)

Table 1. Specification of intercity bus

\subsection{The virtual intercity bus model}

According to the virtual model of intercity bus model, the multi-body dynamic model has been carried in MSC ADAMS/Truck tool (MSC software). Table 1 showed the specificaiton of intercity bus model. The virtual model consised of sub-system as following: chassis body, powertrain, steering system, anti-roll bar, wheel model, front and rear suspension system. Table II display the weight distribution of intercity bus model and the distance of CG position from front axle in this study. 
Table 2. The weight distribution of an intercity bus model

\begin{tabular}{|c|c|c|}
\hline Layout & $\begin{array}{c}\text { Distance from } \\
\text { front axle }\end{array}$ & $\begin{array}{c}\text { \% WD } \\
\text { (F/R) }\end{array}$ \\
\hline I & $3,083 \mathrm{~mm}$ & $40 / 60$ \\
\hline II & $2,826 \mathrm{~mm}$ & $45 / 55$ \\
\hline III & $2,570 \mathrm{~mm}$ & $50 / 50$ \\
\hline
\end{tabular}

\subsection{Steady state cornering condition}

The vehicle dynamic test under steady state condition was virtually simulated on a total of nine conditions. A three constant cornering radius consisted of $50-\mathrm{m}, 75-\mathrm{m}$, and $100-\mathrm{m}$ was performed in each difference weight distribution. For the test, the vehicle models were maneuvered on the test track at initial longitudinal velocity $12 \mathrm{~km} / \mathrm{h}$ for left turn. The data were carried out by 0.1 interval time up to $30 \mathrm{~km} / \mathrm{h}$ under 50 seconds on the flat track.

\section{Results and discussion}

According to the result, the firstly is investigated the lateral translation or lateral slip $\ddot{y}$ explained in equation 2. Normally, the lateral acceleration rises related the curvilinear motion, because of the change in direction in vehicle rotating frame which should have the value as $\dot{\psi} x(\dot{\psi} x r)$. However, the side slip phenomena occur and lead to instability in the real environment. Figure 3 show the value of lateral slip acceleration $\ddot{y}$ form each layout model, and the result also show that the increased vehicle speed of the bus have the more lateral slip acceleration is arise. Consequently, the bus speed in range of $12-30 \mathrm{~km} / \mathrm{h}$ is taken into consideration.

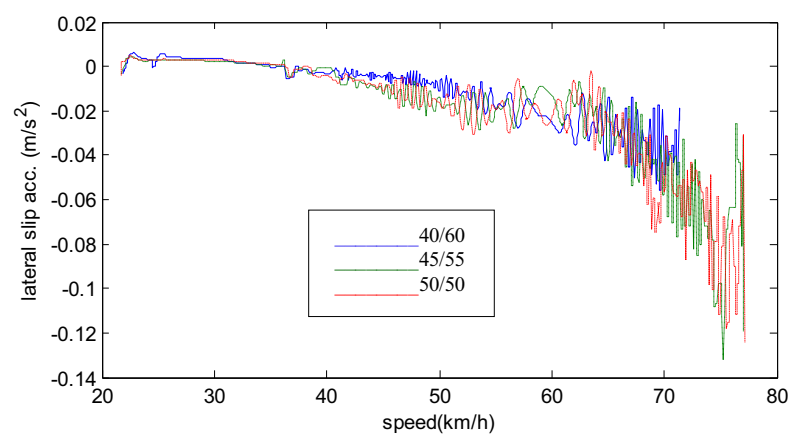

Figure 3. The relationship between lateral slip acceleration and the vehicle speed.

Lateral acceleration versus front wheel side slip is depicted in figure 4. Following equation 4 and 5 the relationship between the steering angle and lateral acceleration is stated and it is shown that the slope of the graph the understeer gradient. But in our cases, constant steering angle input is deployed for three layouts with 75 meter constant turning radius. The steering angle and wheel slip angle are associated and can be approximate via the bicycle model from figure 1 by assuming small rear wheel slip evidenced in figure 5 . The slopes of these are summarized in table 3.

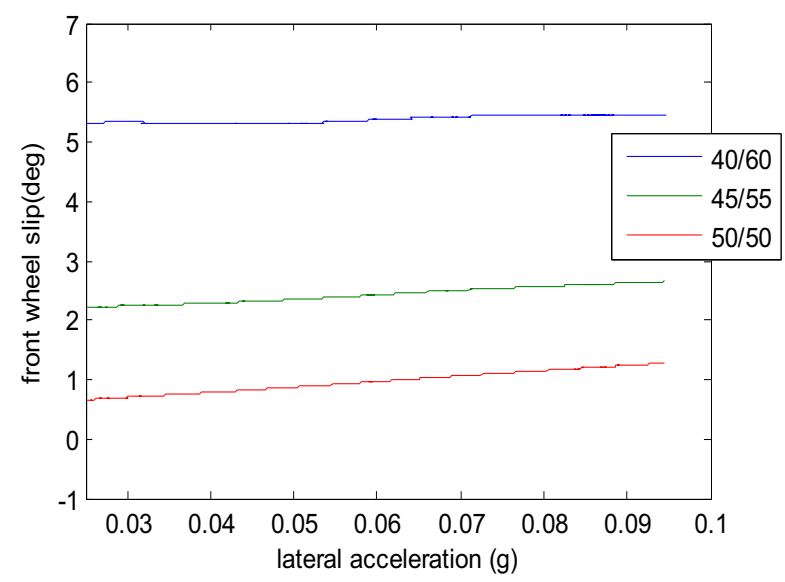

Figure 4. Front wheel slip angle versus lateral acceleration.

Table 3. Understeer gradient of each layout in various turning radius condition

\begin{tabular}{|c|c|c|c|c|}
\hline \multirow{2}{*}{ Layout } & \multirow{2}{*}{$\begin{array}{c}\text { \% WD } \\
\text { (F/R) }\end{array}$} & \multicolumn{3}{|c|}{$\begin{array}{c}\text { Understeer Gradient } \\
\text { (deg/g) }\end{array}$} \\
\cline { 3 - 5 } & & $\mathbf{5 0} \boldsymbol{m}$ & $\mathbf{7 5} \boldsymbol{~}$ & $\mathbf{1 0 0} \boldsymbol{~}$ \\
\hline I & $40 / 60$ & 2.73 & 2.72 & 2.83 \\
\hline II & $45 / 55$ & 6.65 & 6.73 & 6.93 \\
\hline III & $50 / 50$ & 9.2 & 9.04 & 8.98 \\
\hline
\end{tabular}

Table 3 displayed the comparison of weight distribution effective between the various turn radiuses. Clearly, the increase in weight distribution on front axle disclosed the increasing of understeer characteristic. Regarding the magnitude of understeer gradient, the result is closely agree with the N. Yu., et al., [10] which reported the steady state handling behavior of transit bus experimentally. The study revealed that the understeer gradient for the bus left turn was experimentally determined to be $5.1 \mathrm{deg} / \mathrm{g}$. Generally, the understeer gradient of a specific vehicle depend on the several of parameters such as suspension spring stiffness, steering ratio, and wheel alignment conditions, and weight distribution, etc.

In addition, the theoretically understeer gradient is directly proportion to the weight on front axle. That is, the understeer gradient increases with the increase in weight on the front axle. Therefore, with the increase in weight on rear axle, the result exhibit tendency to oversteer behavior $[11,12]$. The trends of study results hold well when compared with the theoretical predictions. Thus, the conclusion can be draw that obtained result are reliable.

Figure 5 showed the comparison of the front slip angle and rear slip angle to confirm the understeer characteristics in each configuration referred to equation 
6. The variation of slip angle of front and rear wheel seems to be the same over the speed range. The slip angle of front wheel increases when the weight distribution is increased toward the front axle
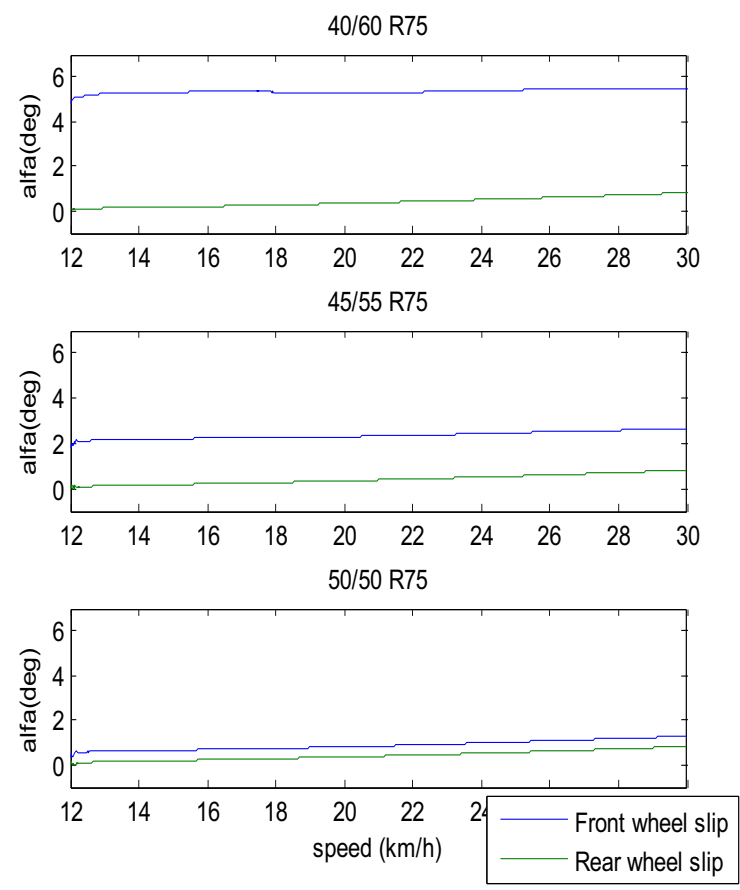

Figure 5. The comparison between front and rear slip in some cases as mentioned.

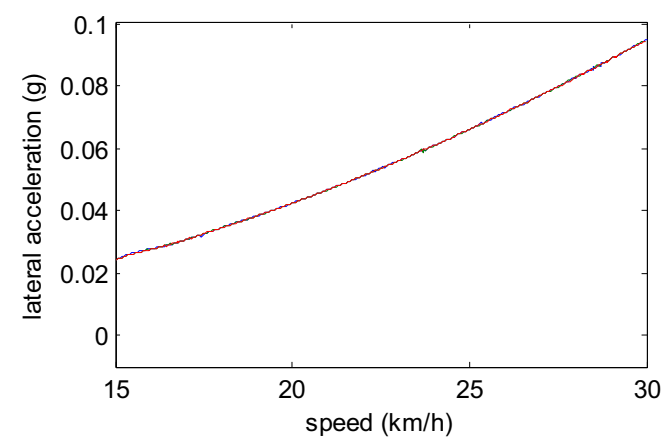

Figure 6. Lateral acceleration determination

One another important parameter ensuring safety during cornering is lateral acceleration as shown in Figure 6 for the worst case. This value is not exceed the threshold value of the heavy duty truck and bus which is approximately less than $0.2 \mathrm{~g}$ for the heavy duty truck mentioned as our experience. Although this threshold seems to be subjectivity, we did perform the experiment in the passenger car and light pick up as reported in [13]. In our simulation range with maximum speed $30 \mathrm{~km} / \mathrm{h}$ during cornering, these values are far from the threshold.

Yaw rate evaluation should be mentioned although it does not show directly the handling stability; it played the important in stability control issue. We tried to compare the rate for the layout I by varying the turning radius. The yaw rate signal just slightly exceeds the desire yaw rate which can be derived from lateral acceleration and the velocity as

$$
\psi_{\text {des }}=\frac{a_{y}}{\sqrt{v_{x}^{2}+v_{y}^{2}}}
$$

We found that yaw rate increases when the radius is smaller and it will increase when it drives faster. The error between simulated and desired one is raised concerning the faster speed. The results from other two cases are in the same trend.

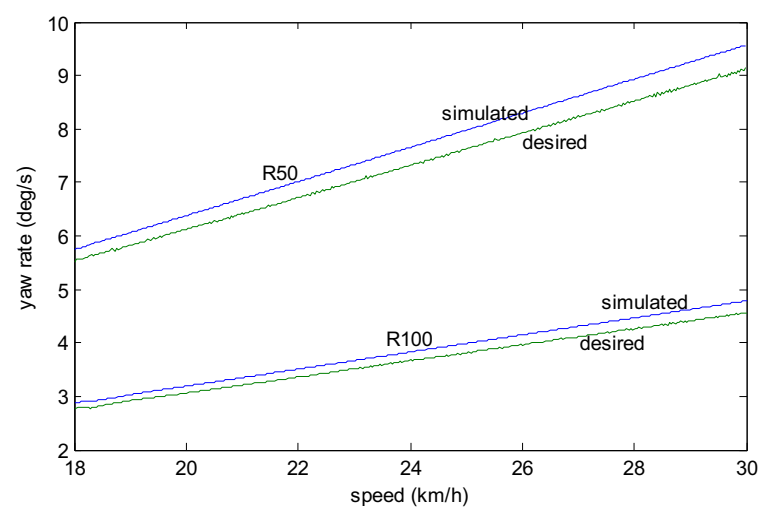

Figure 7. Desired yaw rate and simulated yaw rate in layout $40 / 60$ by varying the turning radius

Theoretically, in curvilinear translation motion the vehicle speed should be in the tangent direction of the travel path or vehicle longitudinal direction, but actually lateral velocity occurs because of side slip. It is obviously that the vehicle moves in an undesired mode. The side slip angle $(\beta)$ is defined as equation 8 .

$$
\beta=\tan ^{-1} \frac{V_{y}}{V_{x}}=\sin ^{-1} \frac{V_{y}}{\sqrt{V_{x}^{2}+V_{y}^{2}}}=\sin ^{-1} \frac{V_{y}}{V} \approx \frac{V_{y}}{V}
$$

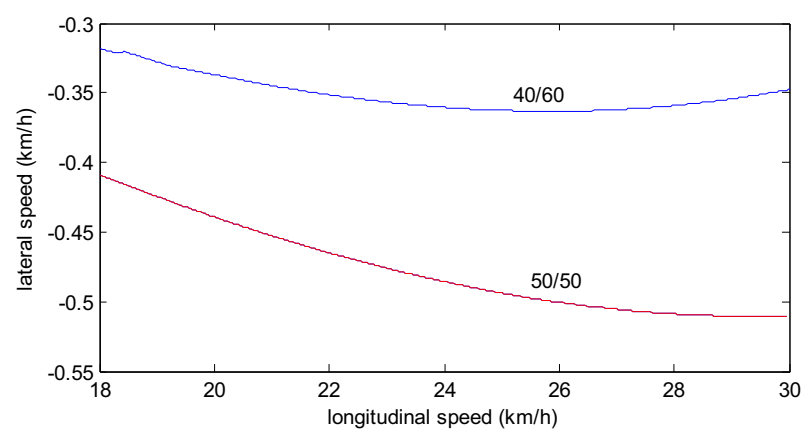

Figure 8. Bus speed in longitudinal and lateral direction using R100.

In our cases of low velocity, mentioned in figure 8 in layout I and III with turning radius $100 \mathrm{~m}$. The lateral speed is near to zero compare to longitudinal one, then the vehicle speed is estimated as the longitudinal speed. In the graph, we can point to side slip angle that layout I has this value less than layout III, consequently, it can be interpreted that model III tends to have more understeer gradient than model I. So that, it is the same way as reported in table 3 . 


\section{Conclusion}

Applying the multi-body dynamic simulation, the effect of weight distribution in each layout with three different turning radiuses to evaluate the vehicle handling characteristic of intercity bus was performed. As the results, the magnitude of understeer gradient increased approximately three times when the weight distribution is increased towards front axle. The variation of slip angle of front and rear tires is responsible for the variation of understeer gradient.

\section{Acknowledgment}

The authors would like to acknowledge Suranaree University of Technology, and also thank National Metal and Materials Center for the sincere support among the research activities of this article.

\section{References}

1. Declare of Department of Land Transport of Thailand, The Government Gazette, 129, 27 (2012)

2. L. Mai, P. Sweatman, Australian Road Research Board, (Rept. No. AIR323-2, 1984)

3. A. Reński, Proc. 24th International Technical Conference on the Enhanced Safety of Vehicles (Gothenburg, Sweden, 2015)

4. L. Prochowski, K. Zielonka, A. Muszyński, J. K Power and Trans, 19, 3 (2012)

5. S. Murthy, M. Gowda, H. Venna, SAE Technical Paper (2016)

6. B. Ayalew, N. Yu, S. Muthiah, B. T. Kulakowski, ASME Int. Mech. Eng Con and Exp, (2004)

7. J. S. Jo, S. H. You, J. Y. Joeng, K. I. Lee, K. Yi, Int. J. of Auto. Tech 9, 5 (2008)

8. H. H. Kim, J. Ryu, Int. J. of Auto. Tech 12, 4 (2011)

9. M. Sherman, G. Myers, SAE Technical Paper (2000)

10. N. Yu, S. Muthiah, B. Kulakowski. $9^{\text {th }}$ International Symposium on Heavy Vehicle Weights and Dimensions (2006)

11. D. Vilela, R. S. Barbosa, J. of the Braz. Soc. of Mech. Sci. and Eng., 33 4, (2011)

12. T. D. Gillespie, Fundamentals of vehicle dynamics, Society of Automotive Engineers (1992)

13. P. Boonporm, Int. $C$ on Science Ecology and Technology I, (Vienna, Austria, 2015) 